\title{
Lossless Immunocytochemistry using Photo-polymerized Hydrogel Thin-films
}

\section{Affiliations:}

${ }^{1}$ Department of Mechanical Engineering, University of British Columbia

${ }^{2}$ Centre for Blood Research, University of British Columbia, Vancouver, BC, Canada

Jeong Hyun Lee ${ }^{1,2}$, Aline T. Santoso ${ }^{1,2,3}$, Emily S. Park ${ }^{1,2,3,4}$, Kerryn Matthews ${ }^{1,2}$, Simon P. Duffy ${ }^{1,2}$ and Hongshen Ma $\mathrm{Ma}^{1,2,3,4^{*}}$

${ }^{3}$ School of Biomedical Engineering, University of British Columbia, Vancouver, BC, Canada

${ }^{4}$ Vancouver Prostate Centre, Vancouver General Hospital, Vancouver, BC, Canada

* Correspondence should be addressed to Hongshen Ma (hongma@mech.ubc.ca)

Short title: Lossless Immunocytochemistry

Immunocytochemistry (ICC), or immunofluorescence microscopy, is an essential biological technique for phenotyping cells in both research and diagnostic applications. Standard ICC methods often do not work well when the cell sample contains a small number of cells $(<10,000)$ because of the significant cell loss that occurs during washing, staining, and centrifugation steps. Cell loss is particularly relevant when working with rare cells, such as circulating tumor cells, where such losses could significantly bias experimental outcomes. In order to eliminate cell loss in ICC protocols, we present a method to encapsulate the cell sample in a photo-polymerized hydrogel thin-film. The hydrogel thin-film is permeable to antibodies and other ICC reagents, thereby allowing the use of standard ICC protocols without modification. The cell sample is physically constrained by the hydrogel at the bottom surface of a standard (unmodified) imaging microtiter plate, thereby enabling the acquisition of high-quality micrographs regardless of the properties of the cell sample or staining reagents. Furthermore, while standard ICC requires several centrifugation steps during staining and washing, our hydrogel encapsulation method requires only a single centrifugation step. This property greatly reduces the time required to perform ICC protocols and is more compatible with robotic platforms. In this study, we show that standard ICC and Cytospin protocols are extremely lossy ( $>70 \%$ loss) when the sample contains less than 10,000 cells, while encapsulating the cells using a permeable hydrogel thin-film results in a lossless ICC process. 


\section{Lossless Immunocytochemistry}

\section{Introduction}

42 Immunocytochemistry (ICC), or immunofluorescence microscopy, is an essential biological assay

43 that uses fluorescence-conjugated antibodies to label cells in order to phenotype them based on

44 protein expression and localization. This assay involves repeated exchange of reagents for cell

45 fixation, permeabilization, blocking, immunostaining, as well as additional buffer washes between

46 each step. When the specimen contains a large number of cells (typically $>10^{5}$ cells per ml), there

47 is sufficient cell density to form a pellet during centrifugation, which enables supernatant removal

48 by pipetting or decanting the fluid. However, when there are fewer cells, the cell density is too low

49 to pellet and many cells may be lost during each supernatant removal step. This issue is particularly

50 important when working with precious samples, where the specimen is limited, or where target

51 cells are rare. For example, detecting circulating tumor cells (CTCs) in the blood of cancer

52 patients $^{1-4}$ or fetal cells in maternal blood ${ }^{5}$, require immunostaining of exceedingly rare cells,

53 where the loss of potential target cells cannot be tolerated.

54 Numerous modifications of the conventional ICC protocol have been developed to prevent cell

55 loss. One approach is to chemically attach cells on a glass slide coated with an adhesive, such as

56 poly-L-lysine, fibronectin, or Cell-tak ${ }^{6-8}$, and then perform the ICC protocol directly on the glass

57 slide. This approach works well for adherent cells grown in culture, but the adhesives are typically

58 ineffective for primary cells or cultured suspension cells. An alternative approach is Cytospin ${ }^{\mathrm{TM}}$,

59 which physically adheres cells to a glass slide using centrifugal force ${ }^{9,10}$. While both primary cells

60 and cells grown in culture can be adhered to a glass slide, this process contributes to significant

61 cell loss. Specifically, when the cell number is relatively small $\left(<10^{5}\right.$ cells per $\left.\mathrm{ml}\right)$, previous studies

62 have reported losses of $>75 \%{ }^{11}$. Additionally, Cytospin is a serial process performed one sample

63 at a time, which significantly limits experimental throughput in screening studies ${ }^{12}$. Finally, while 


\section{Lossless Immunocytochemistry}

64 Cytospin deposits cells in a confined region on a slide, the deposition area typically requires

65 capturing many microscopy fields to image, which adds to the time required for imaging.

66 Therefore, when the sample contains a small number of cells, concentrating cells in a smaller

67 imaging area can significantly reduce imaging time.

68 Here, we present a method to prevent cell loss during ICC by encapsulating cells in a hydrogel

69 thin-film. This approach has been used previously by encapsulating cells in low-melt agarose ${ }^{13}$,

70 which forms a hydrogel matrix that is optically transparent and permeable to ICC reagents.

71 However, this approach has not been widely adopted because the viscosity of agarose solutions

72 which prevent the alignment of cells to a precision surface for imaging. Instead, the agarose

73 hydrogel must be sectioned to image the cells from each optical plane. In this study, we present a

74 cell encapsulation material that has lower density than typical cells in order to enable the alignment

75 of cells by centrifugation to a single layer on the bottom surface of a standard and unmodified

76 imaging microtiter plate. This material is permeable to immunoglobulins, optically transparent

77 with minimal coloration and auto-fluorescence, and mechanically robust to withstand repeated

78 washing. Through additional experiments, we show that the lossless ICC process is able to (i)

79 retain and stain $100 \%$ of the cell sample, (ii) confine the cell sample into a small area for rapid

80 high-quality imaging, and (iii) can be performed with only a single centrifugation step. 


\section{Lossless Immunocytochemistry}

\section{Results and Discussion}

83 Our general approach is to mix cells in a pre-polymer solution that can be crosslinked into a

84 hydrogel upon ultraviolet(UV) light exposure. To enable lossless ICC, the prepolymer solution

85 must be less dense than cells to allow them to sink to the imaging surface via centrifugation. The

86 hydrogel thin film must have sufficient mechanical strength to withstand normal pipetting. Finally,

87 the hydrogel must be sufficiently thin and porous to allow the diffusion of immunoglobulins in a

88 reasonable timeframe $(\sim 1$ hour $)$ (Figure 1$)$.

\section{Density Testing}

90 In order to align cells by centrifugation to a single layer on the bottom glass surface of the imaging

91 microtiter plate, the cell capture solution density must be less than that of typical cells. Given that

92 the lowest density cells are likely to be monocytes, which have a density between 1.067 and 1.077

$93 \mathrm{~g} / \mathrm{ml}^{28}$, the cell capture solution should be less than $1.067 \mathrm{~g} / \mathrm{ml}$ in density. Also, the density must

94 be higher than $1 \mathrm{~g} / \mathrm{ml}$ to sink and encapsulate the cells in bottom plane. We aimed the density of

95 cell capture solution as $1.058 \mathrm{~g} / \mathrm{ml}$.

\section{Mechanical Strength Testing}

97 The mechanical strength of the hydrogel thin-film is important for retaining its structural integrity

98 during pipetting. This property was tested by repeatedly pipetting $40 \mu 1$ of PBS onto the surface of

99 the photopolymerized hydrogel until signs of structure disintegration, such as cracks, tears, and 100 delamination, began to be observable. The polymerized hydrogel thin-film had sufficient 101 mechanical strength to survive $>100$ rounds of repeated pipetting. 


\section{Lossless Immunocytochemistry}

\section{Porosity Testing}

104 Immunoglobulins have an estimated size of $\sim 14 \mathrm{~nm}^{14,15}$. The porosity of hydrogel thin-film must

105 be sufficiently large to permit diffusion of immunoglobulins to the cell sample in a reasonable

106 amount of time. Conventional method for producing macroporous hydrogels include freeze-

107 drying, solvent casting, and gas forming ${ }^{16-21}$. While these methods have been used in tissue

108 engineering applications to produce hydrogels with $>100 \mu \mathrm{m}$ pores ${ }^{22-26}$, these hydrogels have poor

109 mechanical strength and image quality ${ }^{27}$, which makes them incompatible with immunostaining

110 of embedded cells. We evaluated whether ICC could be performed on cells embedded in lossless

111 hydrogel by embedding 22RV1 cancer cells and then using fluorophore-conjugated antibodies to

112 stain the extracellular EpCAM protein, and the intracellular cytokeratin proteins. The $90 \%$ of cells

113 were stained after 1 hour incubation.

\section{Thickness Testing}

115 In addition to porosity, the thickness of the hydrogel thin-film is important for determining the

116 time required for immunoglobulin diffusion. Immunoglobulin stains are introduced on the top

117 surface of the hydrogel and must diffuse to cells located at the bottom surface of the hydrogel,

118 which interfaces with the glass substrate. The thickness of the hydrogel thin-film can be controlled

119 by the UV light intensity and exposure time. Based on the Beer-Lambert law, UV light intensity

120 diminishes exponentially as it penetrates absorbing material. Therefore, UV light applied at the

121 bottom of the imaging plate polymerizes a hydrogel thin-film with thickness directly controlled by

122 exposure time. We tested hydrogel thin-film formation by exposure using a long-wavelength UV

$123 \operatorname{LED}(\lambda=375 \mathrm{~nm})$ for $1,3,5,7$, or 10 seconds. We also tested hydrogel thin-film formation by

124 exposure using standard UV gel imaging system $(\lambda=302 \mathrm{~nm})$ for $5,10,15,20$, and 30 seconds. 


\section{Lossless Immunocytochemistry}

125 The hydrogel thickness was then estimated by first focusing on a cell along the imaging plate

126 surface and then the top hydrogel surface. The z-position of each focal point was obtained from

127 Nikon NIS-BR software and used to estimate the distance between the two points. Three

128 measurements were performed for each experimental condition. Using an UV LED, exposure for

1291 and $3 \mathrm{~s}$ failed to form a hydrogel, while 5, 7, and $10 \mathrm{~s}$ exposures produced hydrogels with

130 thickness of $100+/-20 \mu \mathrm{m}, 500+/-20 \mu \mathrm{m}$, and $1000+/-20 \mu \mathrm{m}$, respectively. Therefore, we

131 selected the $5 \mathrm{~s}$ as the optimal exposure time for UV LED source because it produced a stable

132 hydrogel while minimizing UV exposure and minimizing hydrogel thickness. When using the UV

133 gel imaging system, exposure $<20$ seconds failed to form a hydrogel, while 20 seconds exposure

134 produced $100+/-20 \mu \mathrm{m}$ thickness hydrogel and 30 seconds exposure produced $300+/-20 \mu \mathrm{m}$

135 thickness hydrogel. Therefore, for gel imaging system, we used an exposure time of 20 seconds to

136 minimize hydrogel thickness.

\section{Immunocytochemistry of Hydrogel Encapsulated Cell Samples}

138 To evaluate this cell fixation method for use in immunocytochemistry, we first mixed the cell

139 sample with the prepolymer solution in a glass-bottom imaging well plate and then centrifuged the

140 well plate to align the cells on the surface of the glass. Next, the hydrogel thin-film is formed using

141 a $5 \mathrm{~s}$ UV exposure, at which point, the specimen is immunostained using standard ICC reagents

142 (Figure 2). Since previous efforts to visualize cells in macroporous hydrogels have been limited

143 by image quality ${ }^{27}$, we first evaluated the image quality of hydrogel encapsulated cells by bright

144 field microscopy. While the polymerized hydrogel shows a slight opacity compared to standard

145 buffer, the encapsulated cells were clearly visible under microscopy with no visible degradation

146 in image quality (Figure 3). We then assessed whether the hydrogel impaired immunofluorescence

147 microscopy by staining 22RV1 tumor cells using fluorescence conjugated antibodies specific for 


\section{Lossless Immunocytochemistry}

148 EpCAM and pan-cytokeratin (Figure 4). The cells were clearly visible and the absence of

149 background fluorescence indicates that the unbound antibodies were not adsorbed by the hydrogel,

150 but were efficiently washed away. In order to determine whether fluorescence intensity was

151 hindered by hydrogel encapsulation, we measured the fluorescence intensity of cells from the

152 standard ICC sample and the hydrogel encapsulated sample. We measured the mean fluorescent

153 intensity (MFI) from each cell using a 40×40 pixel square area surrounding each cell. The

154 measured MFI did not show a significant difference between the standard ICC sample and

155 hydrogel encapsulated sample (Figure 5). An important consideration was that all staining

156 parameters were optimized using the standard ICC protocol and the conditions did not require re-

157 optimization for hydrogel encapsulated cells. Thus, cell staining could be achieved under standard

158 conditions in $<2$ hours.

\section{Cell Loss Comparison}

160 We investigate cell loss during ICC resulting from convention protocol, Cytospin, and hydrogel

161 encapsulation. Using 22RV1 prostate cancer cells as a model, we generated a 10-fold dilution

162 series containing 10 to 10,000 cells, and then immunostained the samples using standard ICC,

163 Cytospin, and hydrogel encapsulation. To measure cell loss, we performed triplicate experiments

164 where cells from each specimen were enumerated by two independent reviewers before and after

165 ICC (Figure 6). Cells stained using traditional ICC and Cytospin retained less than 50\% of the

166 cells during immunostaining regardless of the number of starting cells in the sample. In contrast,

167 the hydrogel encapsulated cell sample retained $97-99 \%$ of input cells following immunostaining.

168 The small deviation from ideal likely resulted from incomplete staining rather than cell loss since

169 there are invariably a small fraction of a cell sample that will not stain. The most significant

170 difference in cell loss was observed when fewer than 100 input cells were stained. Under these 


\section{Lossless Immunocytochemistry}

171 situations, almost all (>84\%) cells were lost using standard ICC and Cytospin. In fact, Cytospin

172 failed to retain any cells when there were only 10 cells in the initial sample. Together, these results

173 show that hydrogel encapsulation permits virtually lossless immunostaining that is robust

174 regardless of the starting number of cells in the sample.

\section{Conclusions}

176 This technical note presents a porous hydrogel thin-film for encapsulating cells during

177 immunocytochemistry in order to eliminate cell loss resulting from washing and centrifugation.

178 We show that this hydrogel thin-film is permeable to immunoglobulins, stable enough to withstand 179 pipetting, and allows immunostaining to be performed directly on standard imaging well plates.

180 Compared to standard ICC and Cytospin methods that become highly lossy for small cell samples

$181(<10,000$ cells $)$, this process is lossless and can be used to stain $<10$ cells in a well. Furthermore,

182 this process requires only a single centrifugation step, compared to $>8$ steps for standard ICC, 183 which greatly improves compatibility with robotic systems. Ultimately, this simple and novel 184 application of hydrogels for ICC could greatly improve small cell sample biological assays, such 185 as drug screening on primary cells and identification of rare cells, such as circulating tumor cells. 


\section{Lossless Immunocytochemistry}

\section{Methods}

\section{Chemicals and Hydrogel Preparation}

189 The cell capture imaging reagent (LMR001) was purchased from MilliporeSigma. The

190 paraformaldehyde (PFA), and Tween-20 were purchased from Sigma-Aldrich. Each solution was

191 freshly prepared prior to experiments.

\section{Cell Culture}

193 The cell line 22RV1 (human prostate carcinoma, ATCC CRL2505) was used for immunostaining

194 experiments. This cell line was cultured in RPMI-1640 culture media containing 10\% Fetal Bovine

195 Serum (Gibco) and 1\% penicillin-streptomycin (Gibco) at 5\% CO2 at $37^{\circ} \mathrm{C}$. Cells were re-

196 suspended using $0.25 \%$ Trypsin-EDTA (Gibco), to generate a 10-fold dilution series from $10^{4}-10^{5}$

197 cells per $40 \mu$ l culture media.

\section{Cell Encapsulation}

199 To encapsulate the cells in hydrogel, each cell suspension and $40 \mu \mathrm{L}$ of PBS buffer was first loaded

200 into one well in a 384-high contrast imaging well-plate (Corning). Next, $6.5 \mu \mathrm{L}$ of the cell capture

201 imaging reagent solution is pipetted gently with minimal mixing. The imaging well-plate was then

202 centrifuged for 3 minutes at $3800 \mathrm{rpm}$ (Accuspin 1R, Fisher scientific), and immediately proceeded

203 to next step.

\section{Photo-polymerization}

205 To form a hydrogel thin-film, the previously prepared plate was exposed to UV light using a 375

206 nm UV LED (M375L3, Thorlabs) powered by a LED driver (LEDD1B, Thorlabs), or a cold cathod 


\section{Lossless Immunocytochemistry}

fluorescent lamp (CCFL) UV lamp ( $\lambda=302 \mathrm{~nm}$ ) in a gel imaging system (Gel Doc XR+, Bio-Rad). For UV LED system, the center of the LED was aligned with the center of the each well with 0.5 $\mathrm{mm}$ gap and exposed for 5 seconds under drive current of $700 \mathrm{~mA}$, which provides $470 \mathrm{~mW}$ output

210 power. For the gel imaging system, the location of CCFL UV lamp was pre-marked, and 3 rows

211 of well-plate were aligned on the center of each UV lamp. The exposure was controlled by Image

212 Lab software (Bio-Rad) same as regular DNA gel imaging with a 20 second exposure.

\section{Cytospin Preparation}

214 Cytospin was performed by depositing a $40 \mu \mathrm{L}$ cell suspension directly onto a BSA-coated glass

215 slide using a cytocentrifuge (Cytospin 2, Shadon) at $700 \mathrm{rpm}$ for 3 minutes with low acceleration.

\section{Immunocytochemistry}

217 Immunocytochemistry was performed by first fixing cells in 4\% paraformaldehyde for 10 minutes, 218 washing twice with PBS and permeabilizing the cells with $0.025 \%$ Tween-20 for 15 minutes. After

219 washing the cells twice more with PBS, the cells were blocked with $3 \%$ bovine serum albumin 220 BSA (30 min) and stained for one hour with DAPI $(1 \mu \mathrm{M})$ for DNA, EpCAM-Alexa Fluor 488

221 (1:100 dilution) and Pan-Keratin-Alexa Fluor 647 (1:100 dilution). ICC was performed in parallel

222 on matching samples that were either cytospun onto a glass slide, hydrogel-encapsulated in an

223 imaging plate or non-encapsulated within an imaging plate. This protocol differed between cell

224 specimens because washing of non-encapsulated cells involved adding $40 \mu \mathrm{l}$ of PBS followed by 225 centrifugation (3800 rpm, $3 \mathrm{~min}$ ), washing of cytospin slides employed gentle PBS rinsing and the 226 washing of hydrogel encapsulated cells involved adding PBS and mixing by pipette 10 times.

227 Immunostained cells were directly imaged using both bright field and fluorescent microscopy, 228 using a Nikon Ti-E inverted fluorescent microscope with 10x, 20x and 60x magnification with a 


\section{Lossless Immunocytochemistry}

229 high-resolution camera or a Zeiss laser scanning confocal microscope LSM 780 at 40x

230 magnification.

\section{Cell Counting and Statistical Analysis}

232 Both the initial (prior to plating) and final numbers of all 3 matching ICC samples were manually

233 counted by two individuals from the obtained images using ImageJ software. Experiments were

234 performed 3 times for each cell dilution. Results from the count were averaged and plotted using 235 GraphPad Prism. 
Lossless Immunocytochemistry

237 Acknowledgments: We thank Peter Black for providing the 22RV1 cell line. Jeong Hyun Lee has

238 been supported by the UBC Four Year Fellowship. Emily Park has been supported by the Michael

239 Smith Foundation for Health Research Trainee Award. Kerryn Matthews has been supported by

240 the MITACS Accelerate Postdoctoral Fellowship. This work has been supported by grants from

241 Canadian Institutes of Health Research (312371, 322375, 362500, 381129), Natural Science and

242 Engineering Research Council of Canada (2015-06541, 508392-17), and Prostate Cancer Canada

243 (D2016-1306).

244 Disclosure of Conflicts of Interest: H.M. and J.H.L. are inventors on a patent application

245 describing the hydrogel thin-film technology presented in this manuscript. 


\section{Lossless Immunocytochemistry}

\section{References}

1. de Bono JS, Scher HI, Montgomery RB, et al. Circulating tumor cells predict survival benefit from treatment in metastatic castration-resistant prostate cancer. Clinical cancer research : an official journal of the American Association for Cancer Research. 2008;14(19):6302-9.

2. Cristofanilli M, Budd GT, Ellis MJ, et al. Circulating tumor cells, disease progression, and survival in metastatic breast cancer. The New England journal of medicine. 2004;351(8):78191.

3. Cohen SJ, Punt CJA, Iannotti N, et al. Relationship of circulating tumor cells to tumor response, progression-free survival, and overall survival in patients with metastatic colorectal cancer. Journal of clinical oncology: official journal of the American Society of Clinical Oncology. 2008;26(19):3213-21.

4. Miller MC, Doyle G V, Terstappen LWMM. Significance of Circulating Tumor Cells Detected by the CellSearch System in Patients with Metastatic Breast Colorectal and Prostate Cancer. Journal of oncology. 2010;2010:617421.

5. Hamada H, Arinami T, Kubo T, Hamaguchi H, Iwasaki H. Fetal nucleated cells in maternal peripheral blood: frequency and relationship to gestational age. Human genetics. 1993;91(5):427-32.

6. Masuda H-T, Ishihara S, Harada I, et al. Coating extracellular matrix proteins on a (3aminopropyl)triethoxysilane-treated glass substrate for improved cell culture. BioTechniques. 2014;172(56):172-179.

7. Srinivasan S. A method to fix and permeabilize isolated adult mouse cardiomyocytes for immuno-staining and confocal imaging. Protocol Exchange. 2011;

8. Allen L-AH. Immunofluorescence and confocal microscopy of neutrophils. Methods in molecular biology (Clifton, N.J.). 2014;1124:251-68.

9. Koh CM. Preparation of Cells for Microscopy using Cytospin. Methods in enzymology. 2013;533:235-240.

10. Krishnamurthy V, Satish S, Doreswamy SM, Vimalambike MG. Comparison of Cell Preparations between Commercially Available Filter Cards of the Cytospin with Custom Made Filter Cards. Journal of clinical and diagnostic research : JCDR. 2016;10(7):EC18-20.

11. Dhar M, Pao E, Renier C, et al. Label-free enumeration, collection and downstream cytological and cytogenetic analysis of circulating tumor cells. Nature Publishing Group. 2016;

12. Nakayama T, Mihara K, Kawata J, Kimura H, Saitoh H. Adhesion of suspension cells on a coverslip in serum-free conditions. Analytical Biochemistry. 2014;466:1-3.

13. Vartdal F, Vandvik B, Lea T. Immunofluorescence staining of agarose-embedded cells: A new technique developed for immunological characterization of markers on a small number of cells. Journal of Immunological Methods. 1986;92(1):125-129.

14. Horng Tan Y, Liu M, Nolting B, et al. A Nanoengineering Approach for Investigation and Regulation of Protein Immobilization. ACS Nano. 2008;2(11):2374-2384.

15. Lee J-O, So H-M, Jeon E-K, et al. Aptamers as molecular recognition elements for electrical nanobiosensors. Analytical and Bioanalytical Chemistry. 2008;390(4):1023-1032.

16. Beamish JA, Zhu J, Kottke-Marchant K, Marchant RE. The effects of monoacrylated poly(ethylene glycol) on the properties of poly(ethylene glycol) diacrylate hydrogels used for tissue engineering. Journal of biomedical materials research. Part A. 2010;92(2):441-50. 


\section{Lossless Immunocytochemistry}

17. Van Den Bulcke a I, Bogdanov B, De Rooze N, et al. Structural and rheological properties of methacrylamide modified gelatin hydrogels. Biomacromolecules. 2000;1(1):31-38.

18. Artyukhov AA, Shtilman MI, Kuskov AN, et al. Polyvinyl alcohol cross-linked macroporous polymeric hydrogels: Structure formation and regularity investigation. Journal of NonCrystalline Solids. 2011;357(2):700-706.

19. Gemeinhart R a., Park H, Park K. Pore structure of superporous hydrogels. Polymers for Advanced Technologies. 2000;11(8-12):617-625.

20. Hoffman AS. Hydrogels for biomedical applications. Advanced Drug Delivery Reviews. 2012;64:18-23.

21. Ahmed EM. Hydrogel: Preparation, characterization, and applications: A review. Journal of Advanced Research. 2015;6(2):105-121.

22. Roy TD, Simon JL, Ricci JL, et al. Performance of degradable composite bone repair products made via three-dimensional fabrication techniques. Journal of Biomedical Materials Research. 2003;66A(2):283-291.

23. Kim HJ, Kim U-J, Vunjak-Novakovic G, Min B-H, Kaplan DL. Influence of macroporous protein scaffolds on bone tissue engineering from bone marrow stem cells. Biomaterials. 2005;26(21):4442-4452.

24. Griffon DJ, Sedighi MR, Schaeffer D V., Eurell JA, Johnson AL. Chitosan scaffolds: Interconnective pore size and cartilage engineering. Acta Biomaterialia. 2006;2(3):313-320.

25. Annabi N, Nichol JW, Zhong X, et al. Controlling the porosity and microarchitecture of hydrogels for tissue engineering. Tissue engineering. Part B. 2010;16(4):371-383.

26. Raic A, Rödling L, Kalbacher H, Lee-Thedieck C. Biomimetic macroporous PEG hydrogels as 3D scaffolds for the multiplication of human hematopoietic stem and progenitor cells. Biomaterials. 2014;35(3):929-940.

27. Lee AG, Arena CP, Beebe DJ, Palecek SP. Development of Macroporous Poly(ethylene glycol) Hydrogel Arrays Within Microfluidic Channels. Biomacromolecules. 2010;11(12):3316-3324.

28. Zipursky A, Bow E, Seshadri RS, Brown EJ. Leukocyte density and volume in normal subjects and in patients with acute lymphoblastic leukemia. Blood. 1976;48(3):361 LP - 371. 


\section{Lossless Immunocytochemistry}

\section{Figure Legends}

Fig. 1. Schematic of the hydrogel. (a) cells encapsulated in photo-polymerized hydrogel. (b) the typical hydrogel is impermeable to immunoglobulins. (c) the hydrogel formulated for immunostaining is permeable to immunoglobulins.

324 Fig. 2. The general approach for lossless immunocytochemistry using hydrogel-encapsulated

325 cells. (a) The cell capture solution and cell suspension is added to a standard (unmodified) glass bottom imaging well-plate. The plate is centrifuged to position the cells at the bottom of the plate and the plate is exposed to UV light for $5 \mathrm{~s}$. (b) The supernatant, along with uncured cell capture solution, is removed from the well by pipetting. (c) Immunostaining steps for fixation, permeabilization, immunostaining, as well as multiple washing steps are performed without additional centrifugation steps. (d) Image acquisition can be performed directly on the imaging plate.

Fig. 3. Comparison of bright-field microscopy image of cells encapsulated in hydrogels. (a) before and (b) after photo-polymerization. The polymerized hydrogel shows a slight opacity after photo-polymerization, but there is no significant change in microscopy image quality.

Fig. 4. Micrographs of hydrogel encapsulated cells stained with fluorescent antibodies. (a) $40 \mathrm{X}$ image from a well in a 384 well microtiter plate where $\sim 1,000$ cells are encapsulated in hydrogel and subsequently stained with DAPI (blue), EpCam-Alexafluor-488 (green), PanKeratin-Alexafluor-647 (red). (b-e) Close-ups of merged and separated channels.

Fig. 5. Cell retention comparison. Cell retention following immunostaining of cells using standard ICC, ICC following Cytospin, and hydrogel-encapsulation. Each experiment was performed in triplicate and cells were independently enumerated by two reviewers. Error bars rep resent the standard deviation $(\mathrm{n}=6)$. 


\section{Lossless Immunocytochemistry}

\section{Figures}

(b)

Immunoglobulin (Ig)

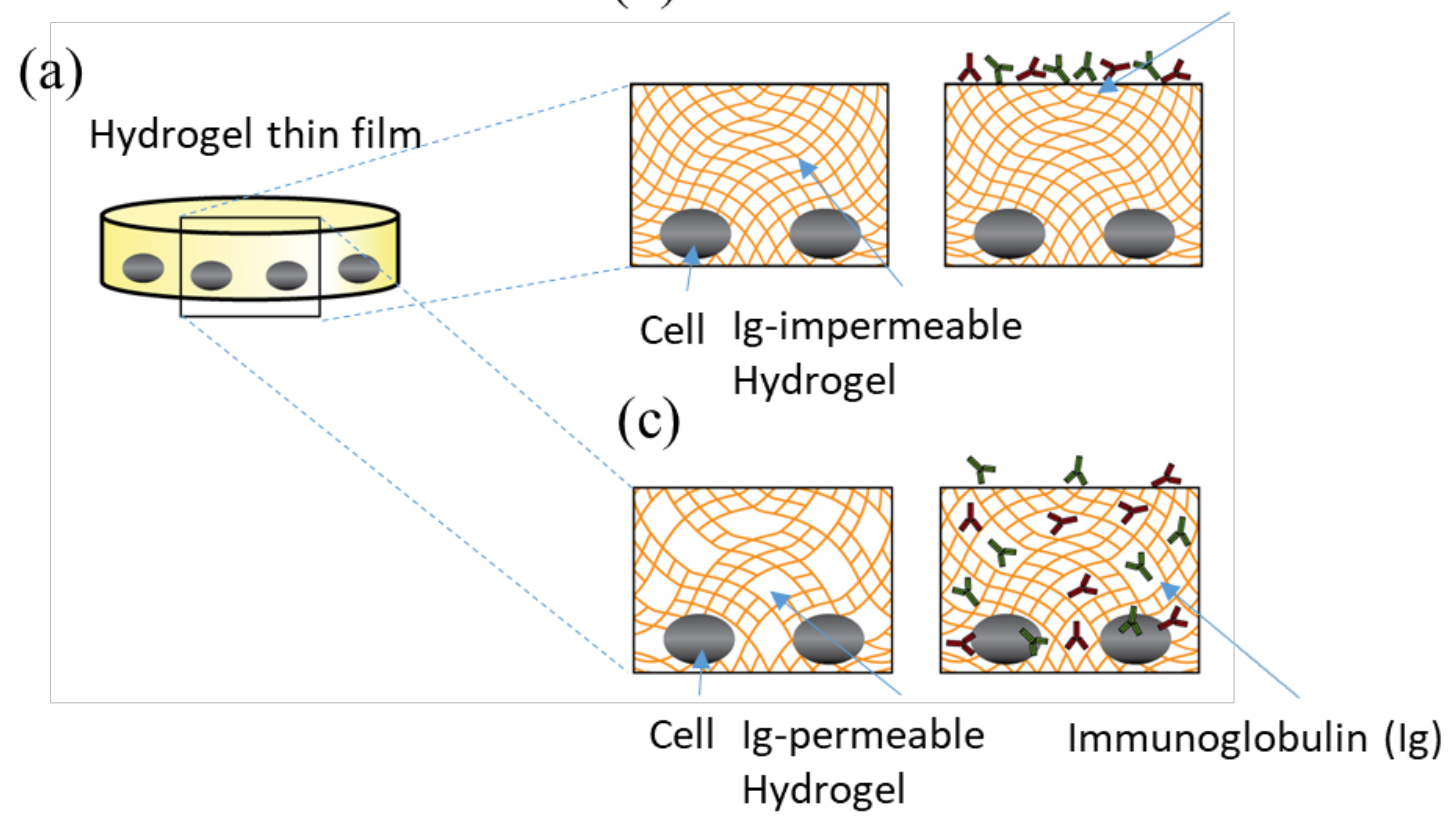

Fig. 1. Schematic of the hydrogel. (a) cells encapsulated in photo-polymerized hydrogel. (b) the typical hydrogel is impermeable to immunoglobulins. (c) the hydrogel formulated for immunostaining is permeable to immunoglobulins. 


\section{Lossless Immunocytochemistry}

(a)

\section{Centrifuged}

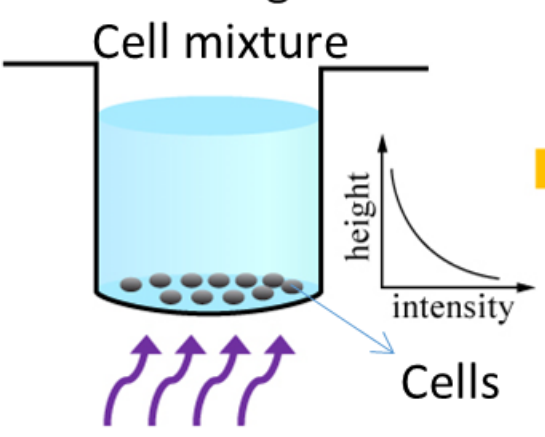

(d)

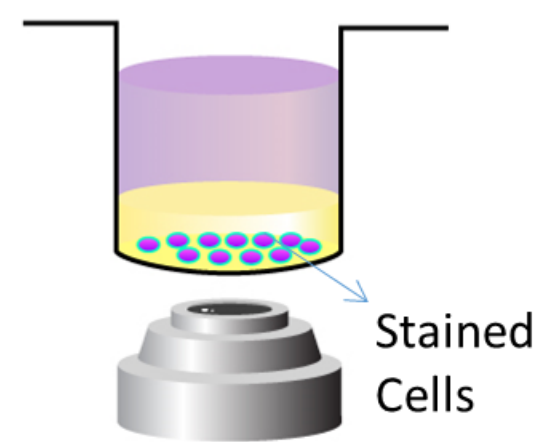

(b)

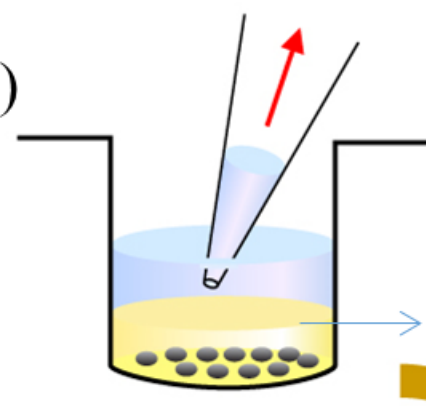

Remove Supernatant

(c)

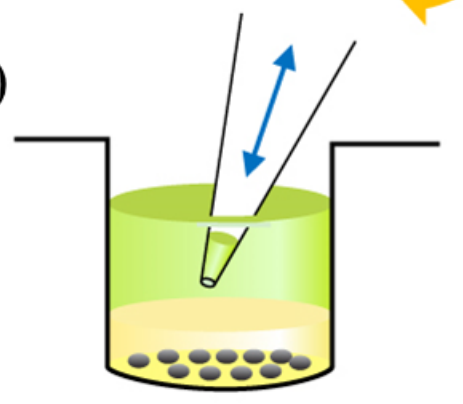

Staining \&

Washing cells. (a) The cell capture solution and cell suspension is added to a standard (unmodified) glass bottom imaging well-plate. The plate is centrifuged to position the cells at the bottom of the plate and the plate is exposed to UV light for $5 \mathrm{~s}$. (b) The supernatant, along with uncured cell capture solution, is removed from the well by pipetting. (c) Immunostaining steps for fixation, permeabilization, immunostaining, as well as multiple washing steps are performed without plate. 


\section{Lossless Immunocytochemistry}

\section{(a)}

(b)

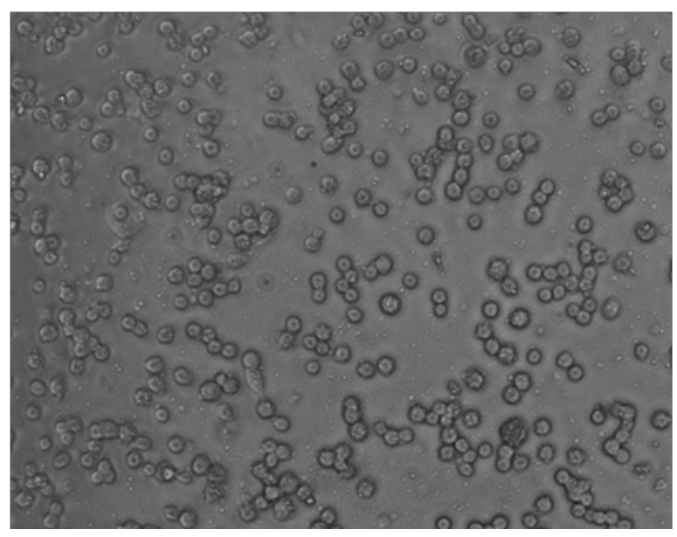

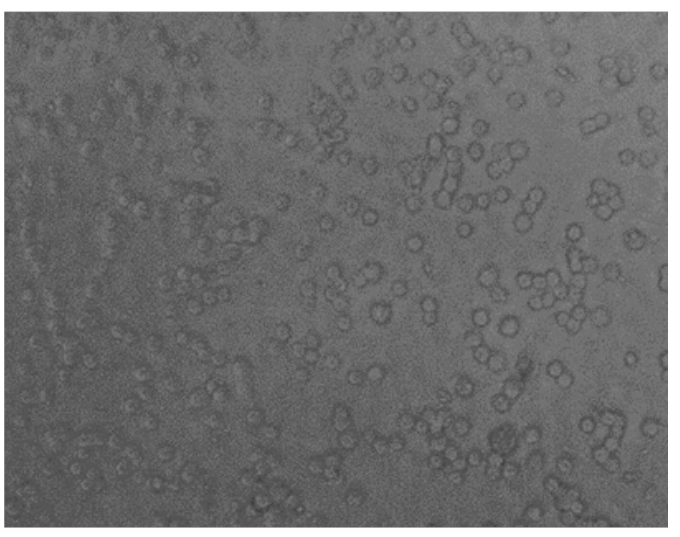

Fig. 3. Comparison of bright-field microscopy image of cells encapsulated in hydrogels. (a) before and (b) after photo-polymerization. The polymerized hydrogel shows a slight opacity after photo-polymerization, but there is no significant change in microscopy image quality. 


\section{Lossless Immunocytochemistry}

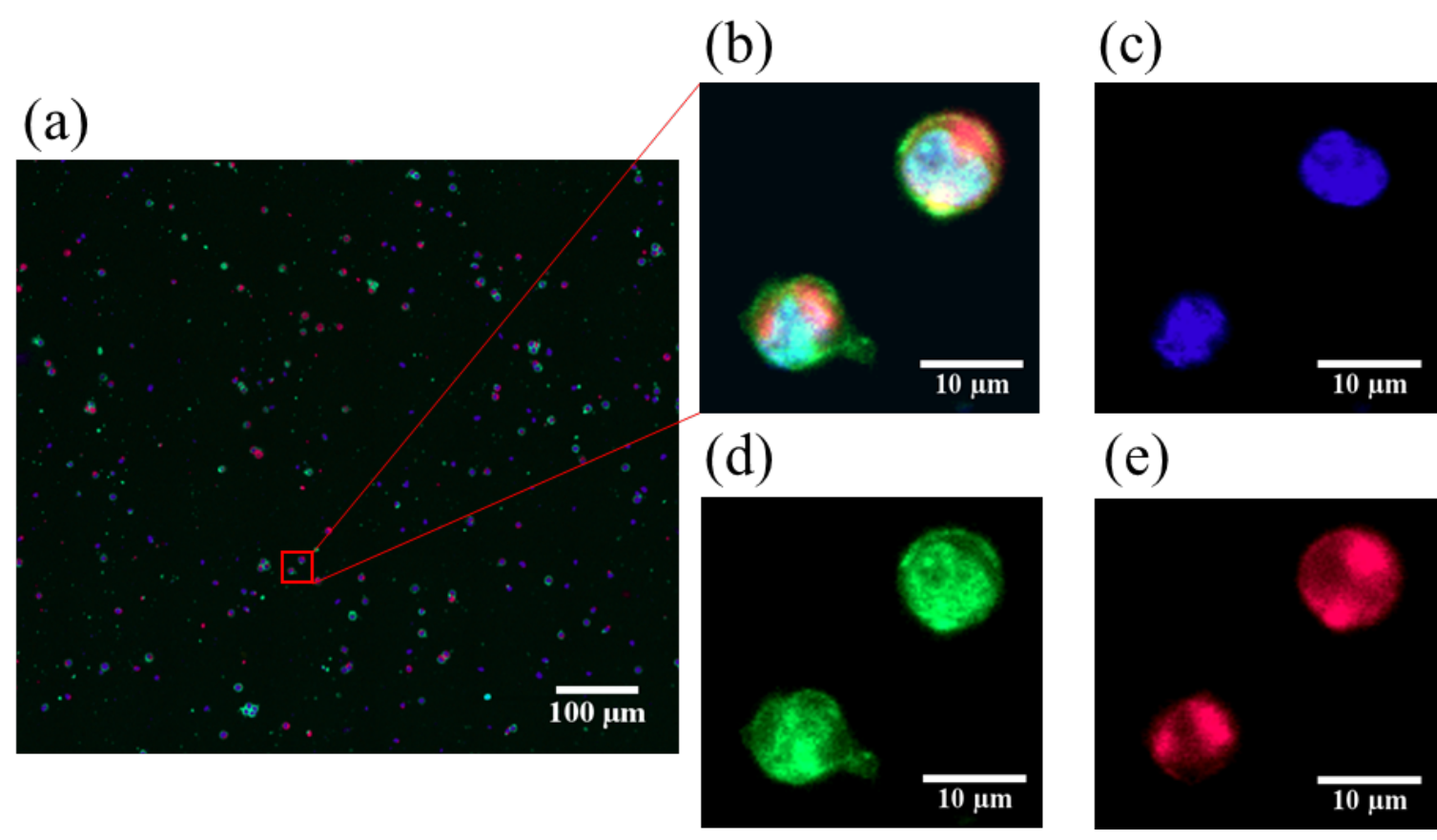

Fig. 4. Micrographs of hydrogel encapsulated cells stained with fluorescent antibodies. (a) $40 \mathrm{X}$ image from a well in a 384 well microtiter plate where $\sim 1,000$ cells are encapsulated in hydrogel and subsequently stained with DAPI (blue), EpCam-Alexafluor-488 (green), PanKeratin-Alexafluor-647 (red). (b-e) Close-ups of merged and separated channels. 
Lossless Immunocytochemistry

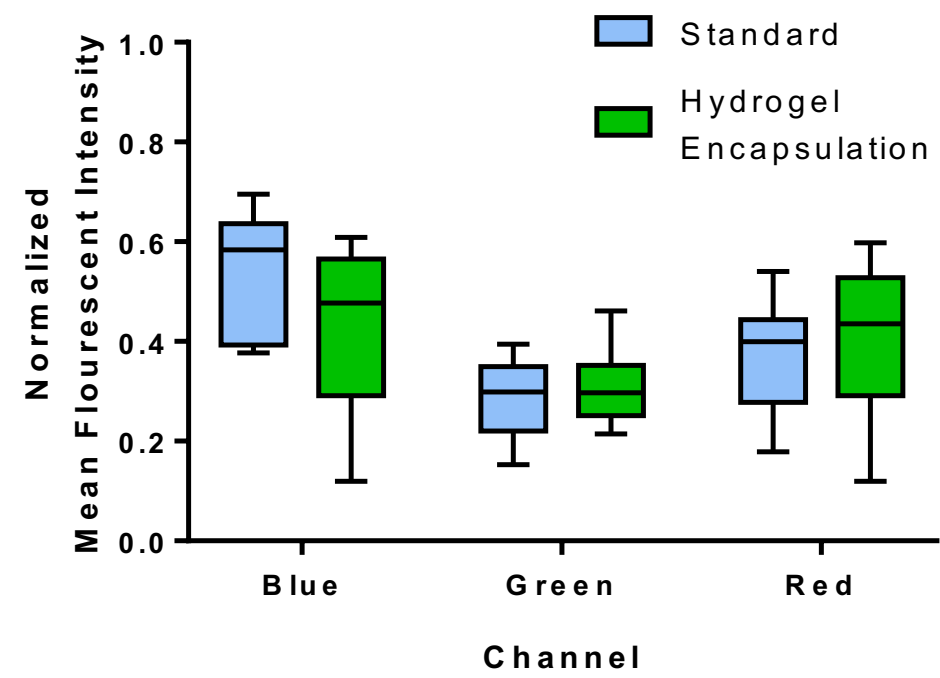

373

374 Fig 5. Comparison of mean fluorescent intensity. The mean fluorescent intensity (MFI) of cells

375 following immunostaining using standard ICC and hydrogel-encapsulation. The MFI was

376 measured from a $40 \times 40$ pixel square window surrounding each cell for each channel. Error bars

377 represent maximum and minimum value $(\mathrm{n}=10)$. 


\section{Lossless Immunocytochemistry}

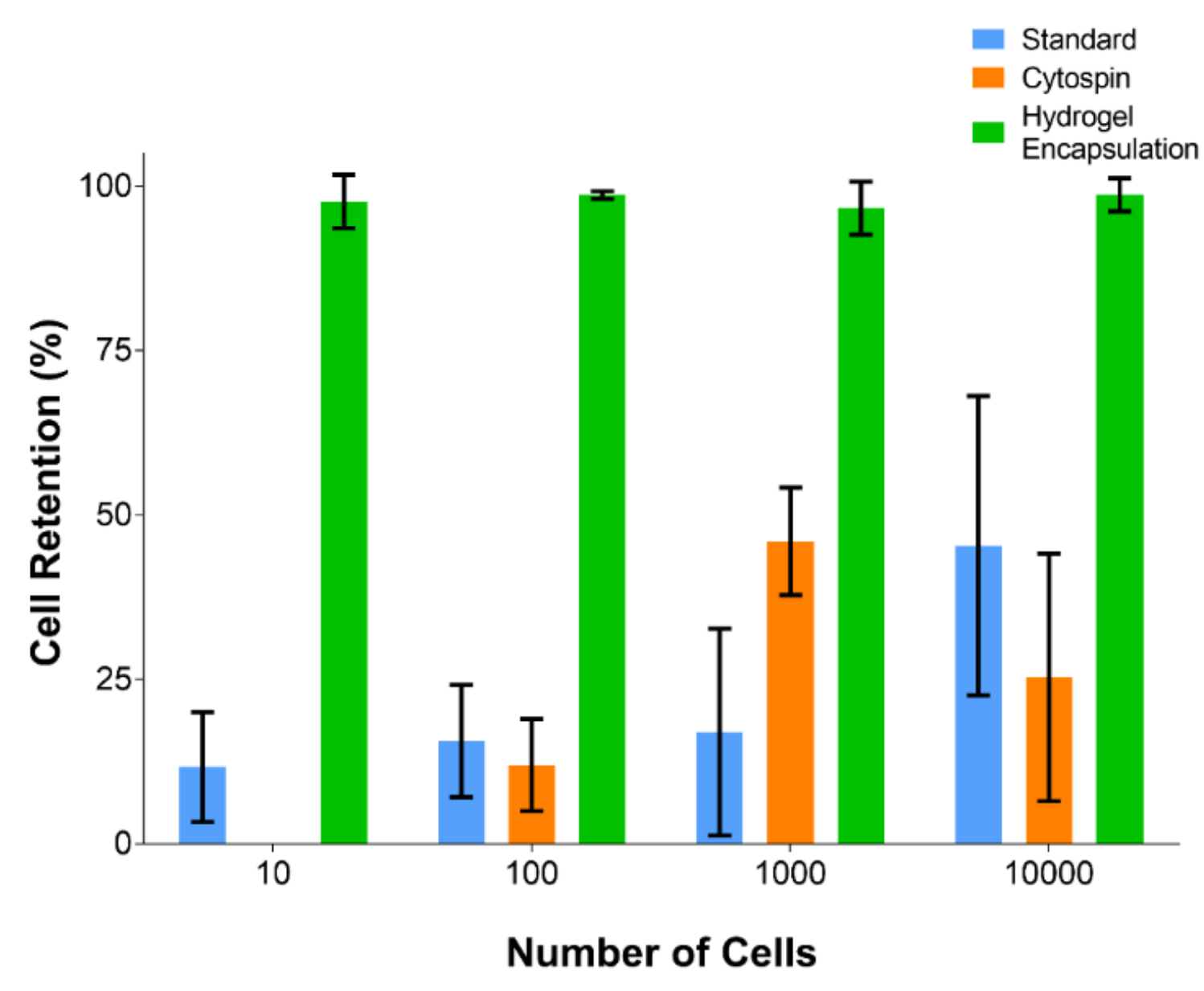

Fig. 6. Cell retention comparison. Cell retention following immunostaining of cells using standard ICC, ICC following Cytospin, and hydrogel-encapsulation. Each experiment was performed in triplicate and cells were independently enumerated by two reviewers. Error bars rep resent the standard deviation $(n=6)$. 